MATHEMATICS OF COMPUTATION

Volume 75 , Number 253, Pages 1-6

S 0025-5718(05)01783-7

Article electronically published on October 21, 2005

\title{
AN OBSERVATION ON KORN'S INEQUALITY FOR NONCONFORMING FINITE ELEMENT METHODS
}

\author{
KENT-ANDRE MARDAL AND RAGNAR WINTHER
}

\begin{abstract}
By utilizing a simple observation on traces of rigid motions we are able to strengthen a result of Brenner (2004) on Korn's inequality for nonconforming finite element methods. The approach here is tightly connected to the theory developed in Brenner's work. Our motivation for the analysis was the desire to show that a robust Darcy-Stokes element satisfies Korn's inequality, and to achieve this the stronger result seems necessary.
\end{abstract}

\section{INTRODUCTION}

In 4 a robust and nonconforming finite element method for a family of singular perturbation problems in two space dimensions was discussed. These problems correspond to linear Stokes-like problems when the perturbation parameter is bounded away from zero, but degenerates to Darcy flow as the parameter is set equal to zero. The discrete velocity fields are chosen as piecewise cubic vector fields with respect to a triangulation of the domain such that the normal component of the fields are continuous over the interelement edges, but the tangential components are only weakly continuous. Hence the space of discrete velocity fields, $\boldsymbol{V}_{h}$, is a subspace of $\boldsymbol{H}$ (div), but not of $\boldsymbol{H}^{1}$.

A key issue related to such nonconforming approximations of $\boldsymbol{H}^{1}$ vector fields is whether Korn's inequality holds for the discrete spaces. Since $\boldsymbol{V}_{h}$ is not a subset of $\boldsymbol{H}^{1}$, this inequality may fail on $\boldsymbol{V}_{h}$. The purpose of this paper is to show that Korn's inequality is indeed true for the finite element space $\boldsymbol{V}_{h}$ introduced in [4. Furthermore, as a by-product of the approach we strengthen a general result of Brenner [1] on Korn's inequality for piecewise $\boldsymbol{H}^{1}$ vector fields.

\section{KoRn's INEQUALITY FOR PIECEWISE $\boldsymbol{H}^{1}$ VECTOR FIELDS}

Let $\Omega$ be a bounded polygonal domain in $\mathbb{R}^{d}, d \geq 1$. Furthermore, $\boldsymbol{L}_{2}=\boldsymbol{L}_{2}(\Omega)$ is the standard space of square integrable vector fields, and the subspace $\boldsymbol{H}^{1}=\boldsymbol{H}^{1}(\Omega)$ is the Sobolev space of vector fields with all first-order derivatives in $\boldsymbol{L}^{2}$. We will use $\|\cdot\|_{0}$ to denote the $\boldsymbol{L}_{2}$ norm and $\|\cdot\|_{1}$ the norm on $\boldsymbol{H}^{1}$, while $|\cdot|_{1}$ is the corresponding seminorm derived from all the first-order derivatives.

Received by the editor October 11, 2004 and, in revised form, November 27, 2004.

2000 Mathematics Subject Classification. Primary 65N30, 74S05.

Key words and phrases. Korn's inequality, piecewise $H^{1}$ vector fields, nonconforming finite elements.

(C)2005 American Mathematical Society Reverts to public domain 28 years from publication 
Korn's inequality, which is frequently referred to as Korn's second inequality, states that there is a positive constant $C=C(\Omega)$ such that

$$
|\boldsymbol{u}|_{1} \leq C\left(\|\boldsymbol{\epsilon}(\boldsymbol{u})\|_{0}+\|\boldsymbol{u}\|_{0}\right), \quad \forall \boldsymbol{u} \in \boldsymbol{H}^{1} .
$$

Here, $\boldsymbol{\epsilon}(\boldsymbol{u})=1 / 2\left(\operatorname{grad} \boldsymbol{u}+(\operatorname{grad} \boldsymbol{u})^{T}\right)$ denotes the symmetric gradient of $\boldsymbol{u}$. The kernel of $\boldsymbol{\epsilon}$ in $\boldsymbol{H}^{1}$ is the space of rigid motions,

$$
\boldsymbol{R} \boldsymbol{M}=\left\{\boldsymbol{a}+\boldsymbol{b} \boldsymbol{x}: \boldsymbol{a} \in R^{d} \text { and } \boldsymbol{b} \in S_{d}\right\},
$$

where $S_{d}$ is the space of anti-symmetric $d \times d$ matrices. An equivalent formulation of Korn's inequality, up to a compactness argument, is the statement that

$$
\|\boldsymbol{u}\|_{1} \leq C\|\boldsymbol{\epsilon}(\boldsymbol{u})\|_{0}, \quad \forall \boldsymbol{u} \in \hat{\boldsymbol{H}}^{1},
$$

where $\hat{\boldsymbol{H}}^{1}$ is the orthogonal complement of $\boldsymbol{R} \boldsymbol{M}$ in $\boldsymbol{H}^{1}$.

When we consider Korn's inequality for nonconforming spaces, or for piecewise $\boldsymbol{H}^{1}$ vector fields, the terms $\|\boldsymbol{u}\|_{1}$ and $\|\boldsymbol{\epsilon}(\boldsymbol{u})\|_{0}$ should be replaced by the corresponding broken norms, i.e., the differential operators involved are defined locally on each element. In [3] Falk analyzed Korn's inequality for some nonconforming two-dimensional finite element spaces. In particular, he showed that the celebrated Crouzeix-Raviart element (cf. [2]) did not satisfy such an inequality. The counterexample constructed in [3] is based on composing local rigid motions into a global function which is not rigid. This is possible due to the weak continuity required for functions in the Crouzeix-Raviart space.

On the other hand, Brenner 1 recently established that a sufficient criteria for Korn's inequality on nonconforming spaces is

$$
\int_{f}[\boldsymbol{u}]_{f} \cdot \boldsymbol{l} d x_{f}=0, \quad \forall f \in F_{h}, \boldsymbol{l} \in\left(\mathbb{P}_{1}(f)\right)^{d} ;
$$

cf. 1, Theorem 3.1]. Here $F_{h}$ is the set of $d$-1-dimensional interelement faces of the mesh, $\left(\mathbb{P}_{1}(f)\right)^{d}$ is the space of linear functions on $f$ with values in $\mathbb{R}^{d}$, and $[\boldsymbol{u}]_{f}$ denotes the jump of $\boldsymbol{u}$ across the face $f$. This criteria is not met by the CrouzeixRaviart space. The weak continuity condition of this space only requires that (2) holds for constant test functions, i.e., for $\boldsymbol{l} \in\left(\mathbb{P}_{0}(f)\right)^{2}$.

The nonconforming finite element space $\boldsymbol{V}_{h}$ introduced in [4 is composed of functions which are cubic vector fields with constant divergence on each triangle, and with linear normal component on each edge. On each triangle this is a space of dimension 9, and the degrees of freedom, indicated by Figure 1, consists of the two lowest-order moments of the normal component and zero-order moment of the

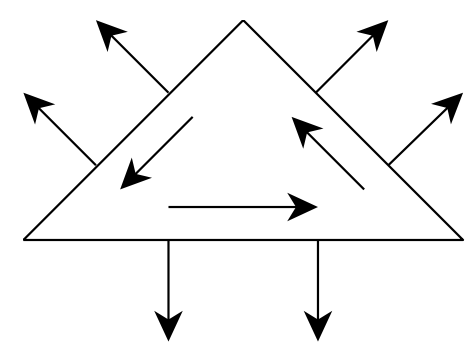

Figure 1. The degrees of freedom of the robust Darcy-Stokes element. 
TABLE 1. Calculated values of $\rho_{h}$ for decreasing values of $h$.

\begin{tabular}{|c|c|c|c|c|c|}
\hline$h$ & $\frac{1}{2}$ & $\frac{1}{4}$ & $\frac{1}{8}$ & $\frac{1}{16}$ & $\frac{1}{24}$ \\
\hline$\rho_{h}$ & 1.94 & 3.56 & 4.40 & 4.71 & 4.78 \\
\hline
\end{tabular}

tangential component of the vector fields on each edge. Hence, the vector fields in $\boldsymbol{V}_{h}$ do not satisfy the criteria (2). On the other hand, if $\boldsymbol{u} \in \boldsymbol{V}_{h}$ is composed of local rigid motions, then the tangential components of $\boldsymbol{u}$ is constant on each edge, and hence $\boldsymbol{u}$ is continuous. Therefore $\boldsymbol{u} \in \boldsymbol{R} \boldsymbol{M}$, i.e., $\boldsymbol{u}$ is a global rigid motion. This shows that a counterexample of the form given in 3] for the Crouzeix-Raviart element cannot be constructed in this case.

To investigate further if Korn's inequality holds or not for the space $\boldsymbol{V}_{h}$, we perform a numerical experiment with the domain $\Omega$ taken as the unit square. We estimate

$$
\rho_{h}=\sup _{0 \neq \boldsymbol{u} \in \boldsymbol{V}_{h}}\|\boldsymbol{u}\|_{1} /\left(\|\boldsymbol{\epsilon}(\boldsymbol{u})\|_{0}^{2}+\|\boldsymbol{u}\|_{0}^{2}\right)^{1 / 2}
$$

for different meshes. Define an operator $A_{h}: \boldsymbol{V}_{h} \mapsto \boldsymbol{V}_{h}$ by

$$
\left(A_{h} \boldsymbol{u}, \boldsymbol{v}\right)_{1}=(\boldsymbol{\epsilon}(\boldsymbol{u}), \boldsymbol{\epsilon}(\boldsymbol{v}))+(\boldsymbol{u}, \boldsymbol{v}), \quad \forall \boldsymbol{u}, \boldsymbol{v} \in \boldsymbol{V}_{h},
$$

where $(\cdot, \cdot)$ and $(\cdot, \cdot)_{1}$ denote the inner products of $\boldsymbol{L}^{2}$ and $\boldsymbol{H}^{1}$, respectively. Then

$$
\rho_{h}=\sqrt{\kappa\left(A_{h}\right)},
$$

where $\kappa\left(A_{h}\right)$ is the condition number of $A_{h}$. The counterexample for the CrouzeixRaviart element given in 3 utilized grids with a particular structure. The simplest example of such a grid is shown in the leftmost picture in Figure 2. Our numerical experiments are also on such grids. In Table 1 we give values for $\rho_{h}$, calculated by Matlab, for decreasing values of the mesh parameter $h$, referring to the spacing on the axis. The results seems to indicate that $\rho_{h}$ is bounded independently of the mesh size.

These computational results indicate that Korn's inequality is valid for this element even if it does not satisfy condition (2). This has led us to re-examine
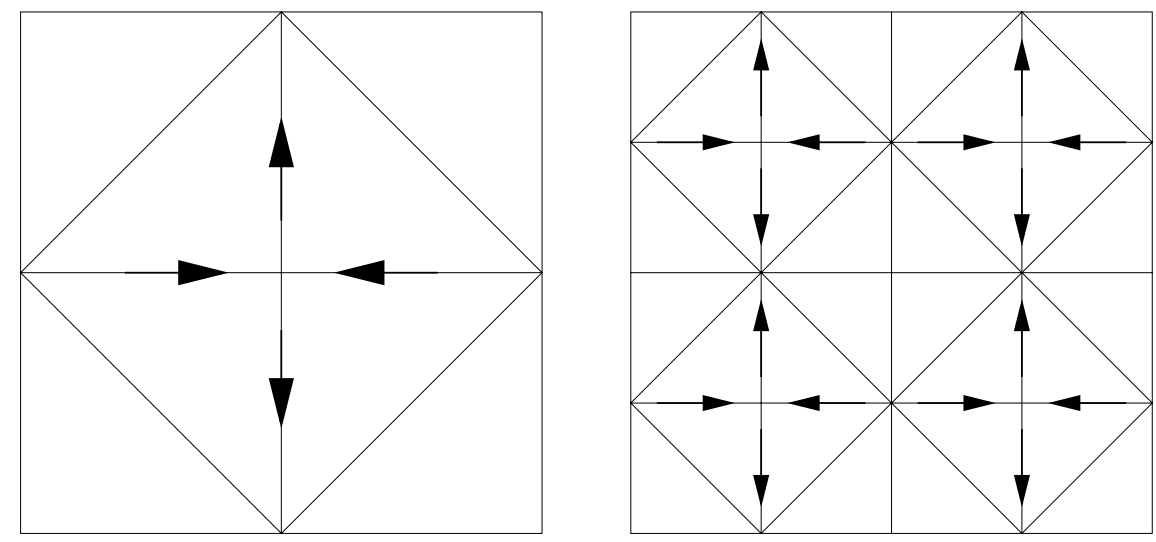

Figure 2. Plot of the two coarsest grids with some of the local rigid motions that are possible for the Crouzeix-Raviart elements. 
Brenner's argument. By using her approach, but by utilizing the fact that the tangential component of traces of rigid motions corresponds to $d$-1-dimensional rigid motions on the interfaces, and not full linears, we have been able to strengthen her result. Basically, we show that it is sufficient that

$$
\int_{f}[\boldsymbol{u}] \cdot \boldsymbol{l} d s=0, \quad \forall f \in F_{h}, \boldsymbol{l} \in \boldsymbol{P}_{1,-}(f),
$$

where $\boldsymbol{P}_{1,-}(f)$ is defined by

$$
\boldsymbol{P}_{1,-}(f)=\left\{\boldsymbol{l} \in\left(\mathbb{P}_{1}(f)\right)^{d}: \boldsymbol{l}_{t} \in \boldsymbol{R} \boldsymbol{M}(f)\right\}
$$

where $\boldsymbol{l}_{t}$ denote the tangential component of the vectorfield $\boldsymbol{l}$. In two space dimensions this means that the normal direction is continuous with respect to linear polynomials, whereas continuity with respect to constants is sufficient in the tangential direction. The element in [4 has this property. In three space dimensions the number of required continuity conditions is reduced from nine to six conditions on each interface.

\section{The MAIN RESUlT}

In this section we state our main result, which is a strengthened version of Theorem 3.1 in [1].

Let $\left\{\mathcal{T}_{h}\right\}$ be a shape regular family of simplicial triangulations of $\Omega$, where $h$ is a parameter indicating the mesh size. If $T \in \mathcal{T}_{h}$ is a simplex the projection operator $\Pi_{T}$ from $\boldsymbol{H}^{1}(T)$ onto $\boldsymbol{R} \boldsymbol{M}(T)$ is defined by

$$
\begin{aligned}
\int_{T} \boldsymbol{u}-\Pi_{T} \boldsymbol{u} d x & =0, \quad \forall \boldsymbol{u} \in \boldsymbol{H}^{1}(T), \\
\int_{T} \operatorname{curl}\left(\boldsymbol{u}-\Pi_{T} \boldsymbol{u}\right) d x & =0, \quad \forall \boldsymbol{u} \in \boldsymbol{H}^{1}(T),
\end{aligned}
$$

where curl $\boldsymbol{u}$ is the antisymmetric part of the gradient of $\boldsymbol{u}$. Following [1] this projection operator has the approximation properties

$$
\begin{array}{r}
\left|\boldsymbol{u}-\Pi_{T} \boldsymbol{u}\right|_{1, T} \leq C\|\boldsymbol{\epsilon}(\boldsymbol{u})\|_{0, T}, \\
\left\|\boldsymbol{u}-\Pi_{T} \boldsymbol{u}\right\|_{0, T} \leq C h_{T}\left|\boldsymbol{u}-\Pi_{T} \boldsymbol{u}\right|_{1, T},
\end{array}
$$

where $h_{T}$ is the diameter of $T$. The corresponding global projection operator $\Pi=$ $\Pi_{h}$ is defined as

$$
\left.(\Pi \boldsymbol{u})\right|_{T}=\Pi_{T} \boldsymbol{u}, \quad \forall T \in \mathcal{T}_{h}
$$

Let the space $\boldsymbol{H}_{h}^{1}$ be defined by

$$
\boldsymbol{H}_{h}^{1}=\left\{\boldsymbol{u} \in \boldsymbol{L}_{2}(\Omega):\left.\boldsymbol{u}\right|_{T} \in \boldsymbol{H}^{1}(T), \quad \forall T \in \mathcal{T}_{h}\right\},
$$

with corresponding norm

$$
\|\boldsymbol{u}\|_{1, h}=\left(\sum_{T \in \mathcal{T}_{h}}\|\boldsymbol{u}\|_{1, T}^{2}\right)^{1 / 2} .
$$


The broken seminorm $|\cdot|_{1, h}$ is defined similarly. We will also need the fact (cf. [1, Lemma 2.2]) that for all piecewise linear vector fields,

$$
|\boldsymbol{u}|_{1, h}^{2} \leq C\left(\|\boldsymbol{\epsilon}(\boldsymbol{u})\|_{0}^{2}+\|\boldsymbol{u}\|_{0}^{2}+\sum_{f \in F\left(\mathcal{T}_{h}\right)} h_{f}^{d-2} \sum_{p \in \mathcal{V}(f)}\left|[\boldsymbol{u}]_{f}^{2}(p)\right|\right),
$$

where $F\left(\mathcal{T}_{h}\right)$ is the set of $d$-1-dimensional interelement faces of $\mathcal{T}_{h}, \mathcal{V}(f)$ is the set of vertices on the face $f$, and $h_{f}$ is the diameter of $f$. Furthermore, $[\boldsymbol{u}]_{f}(p)$ is the jump in $\boldsymbol{u}$ at vertex $p$ across the face $f$. Finally, $\pi_{f}: \boldsymbol{L}^{2}(f) \mapsto \boldsymbol{P}_{1,-}(f)$ is the $L^{2}$ projection.

Our main result is the following theorem which shows, in particular, that the robust Darcy-Stokes element satisfies Korn's inequality.

Theorem 3.1. There exists a constant $C$, depending on the domain $\Omega$ and the shape regularity of the triangulation, such that

$$
|\boldsymbol{u}|_{1, h}^{2} \leq C\left(\|\boldsymbol{\epsilon}(\boldsymbol{u})\|_{0}^{2}+\|\boldsymbol{u}\|_{0}^{2}+\sum_{f \in F\left(\mathcal{T}_{h}\right)} h_{f}^{-1}\left\|\left[\pi_{f} \boldsymbol{u}\right]\right\|_{0, f}^{2}\right), \quad \forall \boldsymbol{u} \in \boldsymbol{H}_{h}^{1} .
$$

Proof. By using the decomposition

$$
|\boldsymbol{u}|_{1, h} \leq|\boldsymbol{u}-\Pi \boldsymbol{u}|_{1, h}+|\Pi \boldsymbol{u}|_{1, h},
$$

and the fact that $\Pi \boldsymbol{u}$ is piecewise linear with $\boldsymbol{\epsilon}(\Pi \boldsymbol{u})=0$, we obtain from (44) and (6) that

$$
|\boldsymbol{u}|_{1, h}^{2} \leq C\left(\|\boldsymbol{\epsilon}(\boldsymbol{u})\|_{0}^{2}+\|\Pi \boldsymbol{u}\|_{0}^{2}+\sum_{f \in F\left(\mathcal{T}_{h}\right)} h_{f}^{d-2} \sum_{p \in \mathcal{V}(e)}|[\Pi \boldsymbol{u}](p)|^{2}\right) .
$$

Furthermore, from (4) and (5), we obtain

$$
\|\Pi \boldsymbol{u}\|_{0} \leq\|\boldsymbol{u}-\Pi \boldsymbol{u}\|_{0}+\|\boldsymbol{u}\|_{0} \leq C\left(\|\boldsymbol{\epsilon}(\boldsymbol{u})\|_{0}+\|\boldsymbol{u}\|_{0}\right) .
$$

Hence, it only remains to estimate the jump terms in (7).

Fix a face $f \in F\left(\mathcal{T}_{h}\right)$. Since $[\Pi \boldsymbol{u}]_{f}$ is a linear function on $f$ and $\pi_{f}[\Pi \boldsymbol{u}]_{f}=[\Pi \boldsymbol{u}]_{f}$, we have

$$
\begin{aligned}
\left|[\Pi \boldsymbol{u}]_{f}(p)\right|^{2} & \leq 2\left(\left|\pi_{f}[\boldsymbol{u}]_{f}(p)\right|^{2}+\left|\pi_{f}[\boldsymbol{u}-\Pi \boldsymbol{u}]_{f}(p)\right|^{2}\right) \\
& \leq C h_{f}^{1-d}\left(\left\|\pi_{f}[\boldsymbol{u}]_{f}\right\|_{0, f}^{2}+\left\|\pi_{f}[\boldsymbol{u}-\Pi \boldsymbol{u}]\right\|_{0, f}^{2}\right) .
\end{aligned}
$$

Finally, utilizing the trace theorem with proper scaling, (4) and (5), we obtain

$$
\begin{aligned}
\left\|\pi_{f}[\boldsymbol{u}-\Pi \boldsymbol{u}]_{f}\right\|_{0, f}^{2} & \leq\left\|[\boldsymbol{u}-\Pi \boldsymbol{u}]_{f}\right\|_{0, f}^{2} \\
& \leq C \sum_{T \in M(f)}\left(h_{f}\left|\boldsymbol{u}-\Pi_{T} \boldsymbol{u}\right|_{1, T}^{2}+h_{f}^{-1}\left\|\boldsymbol{u}-\Pi_{T} \boldsymbol{u}\right\|_{0, T}^{2}\right) \\
& \leq C h_{f} \sum_{T \in M(f)}\|\boldsymbol{\epsilon}(\boldsymbol{u})\|_{0, T}^{2} .
\end{aligned}
$$


Here $M(f)$ is the set of the two simplexes meeting $f$, and we have used that $h_{T} / h_{f}$ is bounded for $T \in M(f)$ by shape regularity. Hence, we conclude that

$$
\sum_{f \in F\left(\mathcal{T}_{h}\right)} h_{f}^{d-2} \sum_{p \in \mathcal{V}(e)}|[\Pi \boldsymbol{u}](p)|^{2} \leq C\left(\|\boldsymbol{\epsilon}(\boldsymbol{u})\|_{0}^{2}+\sum_{f \in F\left(\mathcal{T}_{h}\right)} h_{f}^{-1}\left\|\left[\pi_{f} \boldsymbol{u}\right]\right\|_{0, f}^{2}\right),
$$

and together with (7) this implies the desired inequality.

\section{ACKNOWLEDGMENTS}

The authors are grateful to Professors R. S. Falk and S. Turek for useful discussions on this topic.

\section{REFERENCES}

[1] S. C. Brenner, Korn's inequalities for piecewise $H^{1}$ vector fields, Math. Comp. 73 (2004), 1067-1087. MR2047078 (2005c:65096)

[2] M. Crouzeix and P. A. Raviart, Conforming and non-conforming finite element methods for solving the stationary Stokes equations, RAIRO Anal. Numér. 7 (1973), 33-76. MR0343661 $(49: 8401)$

[3] R. S. Falk, Nonconforming Finite Element Methods for the Equations of Linear Elasticity, Math. Comp. 57 (1991), 529-550. MR.1094947(92a:65290)

[4] K.A. Mardal, X.-C. Tai, and R. Winther, A robust finite element method for Darcy-Stokes flow, SIAM J. Numer. Anal. 40 (2002), 1605-1631. MR1950614(2003m:76110)

Department of Scientific Computing, Simula Research Laboratory and Department of Informatics, University of Oslo, P.O. Box 134, 1325 Lysaker, Norway

E-mail address: kent-and@simula.no

Centre of Mathematics for Applications and Department of Informatics, University of Oslo, P.O. Box 1053, Blindern, 0316 Oslo, Norway

E-mail address: ragnar.winther@cma.uio.no 